\title{
Who Will Deliver the Babies? Identifying and Addressing Barriers
}

\author{
William Rayburn, MD, MBA (J Am Board Fam Med 2017;30:402-404.)
}

The policy brief in this $7 A B F M$ issue reinforces the low percentage of new family medicine graduates who intend to perform obstetric deliveries, and that only $7 \%$ of all family physicians currently do so. ${ }^{1}$ These impressions, gathered from the American Board of Family Medicine Family Medicine Certification examination application questionnaire completed by applicants from 2014 to 2016, were also evident from publications by Nesbitt et al, ${ }^{2}$ Tong and colleagues, ${ }^{3}$ and Rayburn and associates, ${ }^{4}$ which span a 30 -year period.

This policy brief and other published reports provide reliable national data about family physicians who perform deliveries. The large sample sizes across years and the comprehensive nature of the self-reported data demonstrate consistent trends with minimal variation. In our prior publication, we noted that family physicians who worked in partnerships or large group practices were most inclined to perform deliveries. ${ }^{4}$ Their desire to provide newborn care was another influencing factor. Most performed deliveries in nonmetropolitan regions, which experience a shortage of practitioners with maintenance of obstetrics training. Most delivered $\leq 25$ babies per year.

Several practice trends continue to affect both family physicians and obstetrician-gynecologists (OB-GYN): (1) more residency graduates pursue subspecialty training than before; (2) a new generation of physicians who place a greater emphasis on work-life balance; (3) changing practice patterns, including more flexible or part-time schedules; and

From the Department of Obstetrics and Gynecology, University of New Mexico School of Medicine, Albuquerque.

Funding: none.

Conflict of interest: none declared.

Corresponding author: William Rayburn, MD, MBA, Division of Maternal Fetal Medicine, Department of Obstetrics and Gynecology, University of New Mexico, MSC10 5580, 1 University of New Mexico, Albuquerque, NM 87131 (E-mail: wrayburn@salud.unm.edu).
(4) more women in the workforce. ${ }^{5,6}$ While numbers of ACGME -accredited Family Medicine residency programs accredited by the Accreditation Council for Graduate Medical Education have grown, OB-GYN programs have declined, from 257 in 1992 to 237 in 2016. In addition, the number of OB-GYN residency positions increased minimally in relation to growth of the US adult female population. ${ }^{5}$ Nearly all OB-GYN programs are located in large metropolitan areas, and their residents are not inclined to train at rural settings.

It used to be that family medicine and OB-GYN residents pursued their general specialty. In a period of rapid growth of medical knowledge, advancement in technology, and emphasis on provider competencies, more is being expected in obstetric care. Over the past 25 years, the number of fellowships in family medicine obstetrics and maternal/child health have increased substantially. ${ }^{7}$

The number of OB-GYNs for the adult female population has declined from metropolitan to micropolitan and to rural counties. ${ }^{8}$ Approximately half of all US counties lack a single OB-GYN, although most of those counties also lack a hospital that provides maternity services. ${ }^{8}$ In contrast to family physicians, OB-GYNs predominantly migrate to counties that are metropolitan or where a smaller percentage of the population lives in poverty, and to states where there is a lower density of OB-GYNs per unit population. ${ }^{9}$

Women of reproductive age (18-44 years) report more frequent visits to either family physicians' or OB-GYNs' offices compared with other specialities. ${ }^{10}$ A "patient-centered medical home" for pregnant women is now more than a concept; it hinges on the central role of office-based health care coordination. ${ }^{5}$ Several examples of expanded maternity-centered modes exist, although they represent a small portion of the different offices at which prenatal care is offered. ${ }^{5}$ Regardless of the type of clinic, it remains essential for family physi- 
cians and OB-GYNs to work closely as a team, especially when treating pregnant women with chronic medical conditions; to minimize redundant cost; and to optimize resource utilization. ${ }^{10}$

The cost of professional liability insurance has the greatest financial impact on a family physician with a limited obstetric practice. Results from the American College of Obstetricians and Gynecologists Survey on Professional Liability, covering 2012 to 2014, indicated that more than one-third of respondents made $\geq 1$ change in their practice as a result of the affordability or availability of insurance, and almost half made $\geq 1$ change to their practice because of the risk or fear of claims or litigation. $^{11}$

Obstetrics is ranked among the "noncontrollable" lifestyle practices particularly among those aged $\leq 50$ years. ${ }^{12}$ The average age at which an OB-GYN quits obstetrics practice is 48 years (and is younger for female providers), and fewer family physicians provide obstetric care as their practices mature. ${ }^{4,5}$ Many in the Millennial generation, born between 1980 and 2000, view their work and professional achievement as being less central to their lives than other professional activities. ${ }^{13}$ Furthermore, physicians burn out (lose control and/or experience conflicting demands on time and a diminishing sense of worth) at rates twice that among working adults, and no medical specialty is immune. ${ }^{5,14}$

On a more positive note, the projected increase in demand for obstetric services will likely be modest. ${ }^{15}$ The addition of qualified nonphysician obstetric providers, working in concert with physicians, would help address those needs. Nurse midwives and advanced practice nurses are filling a vital role in the maternity care workforce, although their practice does not include essential services such as operative intervention or medically complex perinatal care. The small but growing number of OB-GYNs in general practice who work in hospitals ("OB-GYN hospitalists") has grown in the past decade. ${ }^{16}$ Their roles are important in standardizing inpatient care, in providing high-quality care and improving performance while attempting to control costs, and in aiding physicians caring for their patients during labor and delivery.

Expanded use of new technology is a possible strategy for mitigating delay of obstetric services in rural or underserved areas. Based on the literature provided by the Association of American Medical
Colleges Center for Workforce Studies, however, the empirical evidence favoring telemedicine is currently insufficient for obstetrics. ${ }^{17}$

While all family physicians graduate with some maternity care experience, it is currently difficult to determine who is competent to provide this care independently. Conclusions from a recent Family Medicine Maternity Care Summit brought forth a definition of a roadmap for 3 scopes of maternity care practice for family medicine residents that go beyond the minimum 2 months of training. ${ }^{7}$ These scopes of maternity care were categorized as basic, comprehensive, and advanced provisions. This learning collaborative model, using skills and behavior-based competency tools, will provide a platform for national evidence-based standards that can be used to assess competency.

These identified barriers underscore the need for ongoing evaluations about implications of the evolving health care system, especially for practitioners providing obstetric care. No simple solution to this mounting public health problem exists. Examples of strategies to address the barriers would involve the following directions:

1. Standardize residency and fellowship training to more clearly define and track trainee progress in achieving competencies according to the Family Medicine Maternity Care Summit's 3 scopes of maternity care.

2. Expand family medicine obstetrics and maternal/child health fellowship programs.

3. Increase family medicine maternity care through hospital privileges and policies that especially apply where a low volume of obstetrics patients exists.

4. Examine those states and counties where family physicians are more likely to provide obstetric care in order to identify more optimal models that support patient-centered care and collaboration with OB-GYNs and certified nurse midwives (CNMs).

5. Evaluate the role of mentoring and what prompts family physicians to continue performing deliveries.

6. Determine the barriers and facilitators for rural hospitals to provide maternity services (including anesthesia, general surgery) and newborn services, whether such services are conducive to the recruitment and retention of 
family physicians, and how they might attract pregnant women.

7. Provide financial incentives for qualified physicians to provide usually low-volume obstetric services in rural or underserved areas through improved state, federal, and thirdparty reimbursement or coverage of professional liability insurance.

8. Assess the influence of professional liability insurance on family physicians' decision to perform either prenatal care only or deliveries, especially if standards of training and hospital privileges become more clearly defined and uniform.

9. Short of performing deliveries, encourage family physicians to provide routine prenatal and postpartum care at satellite clinics, with linkages for maternal transfers to regional maternity centers.

10. Evaluate different forms of outreach consultation services with OB-GYNs or maternalfetal medicine subspecialists to determine which work best for optimal patient care.

11. Encourage family physicians to maintain OBGYN skills by attending low-cost obstetric and newborn simulation workshops such as Advanced Life Support in Obstetrics at either academic health centers or regional maternity centers.

12. Support OB-GYN hospitalist programs at regional maternity centers to assist family physicians wishing to provide prenatal and postpartum care only and transfer the mothers, and/or to perform deliveries.

To see this article online, please go to: http://jabfm.org/content/ 30/4/402.full.

\section{References}

1. Barreto TW, Eden AR, Petterson S, Bazemore AW, Peterson LE. Intention versus reality: Family medicine residency graduates' intention to practice obstetrics. J Am Board Fam Med 2017;30:405-406.

2. Nesbitt TS, Connell FA, Hart GL, Rosenblatt RA. Access to obstetric care in rural areas: effect on birth outcomes. Am J Public Health 1990;80:814-8.

3. Tong ST, Makaroff LA, Xierali IM, et al. Proportion of family physicians providing maternity care continues to decline. J Am Board Fam Med 2012;25: $270-1$.
4. Rayburn WF, Petterson SM, Phillips RL. Trends in family physicians performing deliveries, 2003-2010. Birth 2014;41:26-32.

5. Rayburn WF. The obstetrician-gynecologist workforce in the United States: facts, figures, and implications, 2017. Washington, DC: ACOG Distribution Center; 2017.

6. Coutinho AJ, Cochrane A, Stelter K, Phillips RL, Peterson LE. Comparison of intended scope of practice for family medicine residents with reported scope of practice among practicing family physicians. JAMA 2015;314:2364-72.

7. Magee SR, Eidson-Ton S, Leeman L, et al. Family medicine maternity care call to action: moving toward national standards for training and competency. Fam Med 2017;49:211-7.

8. Rayburn WF, Klagholz JC, Murray-Krezan C, Dowell LE, Strunk AL. Distribution of American College of Obstetricians and Gynecologists fellows and junior fellows in practice in the United States. Obstet Gynecol 2012;119:1017-22.

9. Xierali IM, Mivet MA, Rayburn WF. Relocation of obstetrician-gynecologists in the United States, 2005-2015. Obstet Gynecol 2017;129:543-50.

10. Petterson SM, Bazemore AW, Phillips RL, Rayburn WF. Trends in office-based care for reproductiveaged women according to physician specialty: a tenyear study. J Womens Health 2014;23:1021-6.

11. Carpentieri AM, Lumalcuri JJ, Show J, Joseph GF. Overview of the 2015 American Congress of Obstetricians and Gynecologists survey on professional liability. Clin Rev 2015;20:1-6.

12. Anderson BL, Hale RW, Salsberg E, Schulkin J. Outlook for the future of obstetrician-gynecologist workforce. Am J Obstet Gynecol 2008;199:88.e1-8.

13. Twenge JM, Campbell SM, Hoffman BJ, Lance CE. Generational differences in work values: leisure and extrinsic values increasing, social and intrinsic values decreasing. J Management 2010;36:1117-42.

14. Keeton K, Fenner DE, Johnson TR, Hayward RA. Predictors of physician career satisfaction, work-life balance, and burnout. Obstet Gynecol 2007;109: 949-55.

15. Dall TM, Chakrabarti R, Storm MV, Elwell EC, Rayburn WF. Estimated demand for women's health services by 2020. J Womens Health 2013;22:643-8.

16. Tessmer-Tuck J, Rayburn W. Roles of ob-gyn hospitalists during changes in the ob-gyn workforce and practice. Obstet Gynecol Clin North Am 2015;42: 447-56.

17. Association of American Medical Colleges (AAMC). The complexities of physician supply and demand. 2017 update: projections from 2015 to 2030. Washington, DC: AAMC; 2017. 